KS. MAREK STOKŁOSA SCJ

Wydział Prawa Kanonicznego

Uniwersytet Kardynała Stefana Wyszyńskiego w Warszawie

\title{
LE COMPETENZE DEL SUPERIORE PROVINCIALE DI UN ISTITUTO RELIGIOSO RIGUARDO AI BENI TEMPORALI E DELLA LORO AMMINISTRAZIONE
}

Sommario: Introduzione. -1 . Responsabilità del superiore provinciale verso i beni della provincia. - 1.1. La subordinazione dei beni. - 1.2. La vigilanza e controllo sull'amministrazione dei beni. -2 . Rapporto tra superiore provinciale ed economo. - 3. Ruolo della commissione provinciale per gli affari economici. - 4. Amministrazione ordinaria. - 5. Amministrazione straordinaria. - 6. Alienazione. - Conclusione.

\section{Introduzione}

Le province in cui si divide un istituto religioso hanno, in virtù del diritto della Chiesa, come afferma il can. $634 \$ 1 \mathrm{CIC/83}$, il diritto di acquistare, possedere, amministrare e alienare, a norma delle Costituzioni, i loro beni temporali per realizzare i propri fini e quelli dell'istituto come tale. A questa struttura che gode di personalità giuridica pubblica è preposto un governo personale nell'ufficio del superiore provinciale il quale, con l'autorità concessa dal diritto comune e da quello proprio, governa tale struttura, composta non solo dai religiosi, ma anche dalle cose materiali chiamate "beni temporali".

La provincia non potrebbe compiere la sua missione nel tempo se non avesse a disposizione i beni necessari. Il superiore provinciale, in quanto responsabile della missione della provincia, è responsabile anche dell'amministrazione dei beni della stessa che sono in 
funzione delle persone e della sua missione. Questa responsabilità lo abilita a compiere, in nome della provincia, gli atti verso i beni ad essa appartenenti, richiesti da una corretta amministrazione, nel pieno rispetto delle leggi promulgate dall'autorità ecclesiastica e di quelle civili, canonizzate dalla Chiesa.

I beni della provincia, in quanto beni ecclesiastici, sono regolati (per quanto riguarda l'acquisto, il possesso, l'amministrazione e l'alienazione) dalle norme del diritto comune, specialmente dalle disposizioni del Libro $\mathrm{V}$ del vigente Codice, completate da alcune norme specifiche per gli istituti religiosi contenute nei cann. 634-640, e dal diritto proprio di ogni istituto, il quale dovrà applicare opportune norme circa l'uso e l'amministrazione dei beni in aggiunta e a specificazione delle norme universali ${ }^{1}$.

\section{Responsabilità del superiore provinciale verso i beni della provincia}

Il superiore provinciale governa una parte immediata dell'istituto, la quale dovrà essere composta, come richiesto dal can. $621 \mathrm{CIC} / 83$, da una pluralità di case religiose canonicamente erette. Dalla definizione di provincia risulta chiaramente che queste case, con tutti i fattori che le compongono, tra cui i loro beni temporali, comprese anche le loro attività e opere, sono soggette all'autorità del superiore provinciale. In concreto, i beni temporali delle case fanno parte dei beni della provincia, anche se a queste case il diritto concede una propria personalità giuridica ${ }^{2}$.

Ai beni della provincia si dovranno aggiungere tutte le strutture di cui la provincia è proprietaria e che gestiscono attività secondo i fini dell'istituto, indipendentemente dalle case religiose canonicamente erette, anche se hanno una propria personalità giuridica riconosciuta dalle leggi civili. Tutte queste strutture rimangono soggette all'autorità

\footnotetext{
${ }^{1}$ Cf. can. $635 \$ 1-2$ CIC/83; J.J. PINEDA, La capacidad económica de institutos, provincias y casas y sus limitaciones, Commentarium pro Religiosis et Missionariis 74(1993), p. 90.

${ }^{2}$ Cf. can. $634 \$ 1 \mathrm{CIC} / 83$.
} 
del superiore provinciale come superiore maggiore nell'ambito dell'amministrazione dei beni. Da tale soggezione sono escluse tutte le attività e opere affidate dal vescovo diocesano o da istituti ecclesiastici. I beni di queste opere o attività appartengono a tali enti e sono amministrati secondo il diritto particolare della rispettiva diocesi e secondo le norme delle convenzioni stipulate. Ad essi si dovrà attenere sia il superiore provinciale, sia l'economo provinciale ${ }^{3}$.

Il superiore provinciale è il primo amministratore dei beni temporali della provincia. Egli può fare non solo ciò che fanno l'economo provinciale e gli altri amministratori dei beni delle attività a lui soggette, ma anche deve rispondere in ultima istanza dell'amministrazione dei beni davanti a Dio e davanti alla Chiesa e all'istituto. Egli, come superiore della persona giuridica pubblica, agisce in piena responsabilità, a nome della provincia, quando compie degli atti di proprietà, stipula o manda in esecuzione dei contratti, o quando compie diversi atti giuridici ${ }^{4}$, secondo le norme della Chiesa e del diritto civile ${ }^{5}$.

${ }^{3}$ Cf. J.L. Gutiérrez, Gli istituti di vita consacrata e le società di vita apostolica come soggetti dell'amministrazione dei beni temporali, in: AA.VV., I beni temporali della Chiesa. XXII Incontro di Studio, Passo della Mendola - Trento, 3 luglio - 7 luglio 1995, Milano 1997, p. 169.

${ }^{4}$ Cf. can. $1279 \$ 1 \mathrm{CIC} / 83$.

${ }^{5} \mathrm{Nel}$ campo dell'amministrazione dei beni, il diritto della Chiesa rimanda alle leggi civili dello Stato, le quali devono essere osservate come se fossero leggi del diritto canonico, se non sono contrarie al diritto divino e se il diritto canonico dispone diversamente. Cf. cann. 22 e 1290 CIC/83. L'ambito di intervento della legge civile riguarda specialmente gli aspetti/concetti di: contratto (cann. 1290; 1574); testamento (can. $668 \$ 1$ ); riconoscimento civile delle istituzioni ecclesiastiche (can. $1274,5^{\circ}$ ); proprietà dei beni ecclesiastici (can. $1284,2^{\circ}-3^{\circ}$ ), donazioni (can. $1297 \$ 2$ ); alienazioni (can. 1293) e vari altri affari giuridici. Cf. J.J. PinedA, La capacidad económica..., op. cit., p. 92; D.J. ANDRÉs, I religiosi datori di lavoro e lavoratori secondo il Codice di diritto canonico, Commentarium pro Religiosis et Missionariis 72(1991), p. 7-12; V. De Paolis, La vita consacrata nella Chiesa, Bologna 1992, p. 249; idem, I beni temporali e la loro amministrazione, in: AA.VV., I religiosi e il nuovo Codice di diritto Canonico, Atti della XXIII assemblea CISM, Roma 1984, p. 140.

Una provincia può avere strutture proprie non solo sul territorio di un'unica nazione, ma addirittura in territori di varie nazioni, per cui nell'amministrazione dei beni si 
Nonostante che nel governo dei beni temporali il superiore provinciale sia affiancato dall'economo provinciale, come prevede il can. $636 \$ 1$, il diritto comune e quello proprio di ogni istituto concedono alcune responsabilità e atti di governo riguardo i beni temporali che possono essere compiuti esclusivamente dal superiore provinciale.

\subsection{La subordinazione dei beni}

In seguito all'applicazione del principio di subordinazione dei beni, definito nel diritto proprio, il superiore maggiore, tra cui il superiore provinciale, può avere il diritto di imporre un contributo ordinario alle persone giuridiche a lui soggette che hanno le risorse per versarlo a scadenze fisse per realizzare i fini della provincia stessa e della condivisione dei beni. Può avere anche, se prevede così il diritto proprio, la facoltà di imporre un contributo straordinario per venire incontro a qualche speciale necessità della provincia o della stessa Congregazione. I motivi dell'applicazione di un contributo speciale possono essere diversi, cominciando, tra l'altro, da una disgrazia che abbia colpito una casa o una provincia, e giungendo fino alla costituzione di un'attività o la ristrutturazione di una casa religiosa o una chiesa, per le quali sia necessario avere molte risorse finanziarie.

Una altra area della subordinazione dei beni delle case religiose all'autorità del superiore provinciale, è l'amministrazione straordinaria e l'alienazione dei beni, nel caso che la natura e il valore di tali beni oltrepassino le competenze dei superiori locali. Lo stesso concetto è valido anche per i superiori provinciali, i quali dovranno rivolgersi al superiore generale quando gli atti i superano le loro competenze; lo stesso superiore generale è subordinato alla Santa Sede, per valori che superano le competenze a lui concesse.

Oltre il concetto della subordinazione dei beni temporali al superiore provinciale, nel diritto proprio potrebbero essere definiti altri affari o atti che riguardano i beni temporali delle singole comunità e della provincia come tale, sarebbero affidati solo al superiore

dovrà tenere conto di questo aspetto, applicando ai singoli casi il diritto civile del rispettivo luogo. Cf. J.L. GutiérRez, Gli istituti di vita consacrata..., op. cit., p. 166. 
provinciale, con o senza un determinato intervento del suo consiglio. Tra questi affari o interventi, oltre quelli definiti nel concetto di amministrazione ordinaria e straordinaria, e alienazione, di cui tratteremo a parte, possono essere previsti alcuni atti di maggior importanza, tra cui: 1) la nomina dell'economo locale e degli altri amministratori delle opere $\mathrm{o}$ attività della provincia; 2 ) la vigilanza sull'amministrazione delle case e delle altre strutture soggette all'autorità del superiore provinciale ${ }^{6}$; 3) la nomina dell'economo provinciale (can. 634 $\$ 1 \mathrm{CIC} / 83)$; 4) accertarsi che ogni persona giuridica della provincia sia riconosciuta dalle autorità civili; 5) concedere l'autorizzazione al superiore locale o all'economo provinciale, di stare in giudizio davanti a un tribunale civile (can. $1288 \mathrm{CIC} / 83$ ).

\subsection{La vigilanza e controllo sull'amministrazione dei beni}

Il superiore provinciale, come abbiamo sopra sottolineato, ha il diritto e dovere di vigilanza e controllo sull'amministrazione dei beni della provincia e delle persone giuridiche a lui soggette. Questa vigilanza comporta, tra l'altro, il dovere di controllare che nell'amministrazione vengano osservate sia le leggi del diritto canonico, sia quelle del diritto civile, e di fornire le indicazioni e le direttive circa l'amministrazione dei beni ${ }^{7}$. Questo controllo viene realizzato tramite le visite e le analisi dei resoconti di amministrazione dei beni della provincia e delle singole comunità. Il superiore provinciale è obbligato a fare, personalmente o tramite un delegato, le visite alle case e alle attività della provincia secondo i modi ed i tempi stabiliti dal diritto proprio. Tra gli aspetti di tale visite, non dovrebbe mai mancare quello della gestione dei beni. Un controllo più specifico può essere effettuato durante la visita dell'economo provinciale, effettuata per mandato del superiore provinciale. I risultati di tale visita amministrativa dovranno essere trasmessi al superiore provinciale e al suo consiglio, con eventuali suggerimenti che dovranno essere

\footnotetext{
${ }^{6}$ Cf. can. 1276 CIC/83.

${ }^{7}$ Cf. V. De Paolis, L’amministrazione dei beni: responsabilità dei superiori e degli economi, Informationes 27/2(2001), p. 115.
} 
applicati nell'amministrazione dei beni delle singole comunità o di altre attività.

Un altro aspetto di vigilanza è l'analisi dei resoconti di amministrazione della provincia, delle comunità e delle singole attività ${ }^{8}$. Ai periodi stabiliti secondo il diritto proprio o dalle disposizioni del superiore provinciale stesso, gli amministratori dei beni devono presentare i resoconti della loro amministrazione. Tra gli amministratori è incluso anche l'economo provinciale, il quale dovrà fornire il resoconto dell'amministrazione dei beni di tutta la provincia ${ }^{9}$. Dopo l'esame del resoconto di tale amministrazione, il superiore provinciale, entro i limiti del diritto universale e proprio, può dare alcune indicazioni speciali, in seguito alle osservazioni critiche circa l'amministrazione dei beni delle persone giuridiche o delle attività soggette alla sua autorità. Nel caso in cui egli rilevi una grave negligenza o mancanza nell'osservanza delle norme del diritto canonico e civile nell'amministrazione dei beni, dovrà prendere verso gli amministratori, adeguati rimedi, non escluda anche la rimozione dall'incarico.

Ultimo aspetto della vigilanza è il bilancio di ogni persona giuridica e delle singole attività della provincia non collegate con le case religiose. Tali bilanci dovranno essere redatti dai rispettivi economi o amministratori, secondo le disposizioni del diritto proprio. Questi bilanci dovranno essere esaminati e approvati dal superiore provinciale con l'intervento del proprio consiglio, precisato nel diritto proprio. Le opere e attività della provincia non collegate con le case religiose, costituite con lo scopo di produrre risorse economiche al fine di mantenere le opere della missione propria dell'istituto, secondo il parere dello scrivente, devono rimanere sotto uno speciale controllo e vigilanza del superiore provinciale. Tra esse ci sono strutture sanitarie, di educazione, di comunicazione sociale, strutture alberghiere. Purtroppo spesso capita, che esse, sfuggendo alla vigilanza della competente autorità della provincia, provocano gravi problemi per la stessa provincia, come proprietaria di tali attività. Succede questo

${ }^{8}$ Cf. can. $636 \$ 2 \mathrm{CIC} / 83$.

${ }^{9}$ Cf. J.J. Pineda, La capacidad económica..., op. cit., p. 109. 
in seguito all'affidamento dell'amministrazione di tali opere esclusivamente a personale laico, senza disporre di mezzi o organismi di controllo e vigilanza; lo stesso può succedere per una sbagliata interpretazione da parte di certi amministratori, i quali pensano che tali attività, in quanto riconosciute dall'autorità dello stato come società o entità rivestite di personalità giuridica civile, siano sottoposte solo alle leggi dello Stato. Gli amministratori di queste opere o attività devono invece comportarsi, non solo secondo le leggi civili, ma anche secondo le leggi canoniche del diritto universale della Chiesa e quello particolare dell'istituto, leggi che per alcuni atti comporta anche limitazioni alle competenze per concedere autorizzazioni, non escludendo addirittura l'intervento della Santa Sede.

Infine, la riflessione sulla responsabilità del superiore provinciale circa i beni temporali della provincia come tale, è necessario affermare che la sua autorità è subordinata, in alcune questioni circa la gestione dei beni, al superiore generale e al suo consiglio. Questa subordinazione o soggezione all'autorità del superiore generale consiste nel fatto che essa può imporre alle province contributi ordinari o straordinari, come anche decidere di trasferire, in caso di necessità, i beni di una provincia ad un'altra. C'è anche una certa subordinazione nell'amministrazione straordinaria e nell'alienazione dei beni temporali. Questi concetti saranno trattati successivamente.

\section{Rapporto tra superiore provinciale ed economo}

Accanto all'ufficio di superiore provinciale, nell'amministrazione dei beni della provincia, il can. $636 \$ 1 \mathrm{CIC} / 83$, affianca la figura dell’economo provinciale, che ha un determinato ruolo, affidato a lui sia dal diritto comune sia da quello proprio, nella gestione dei beni della provincia. Il diritto comune, opportunamente, esige, nel caso della provincia, che l'incarico di economo sia svolto da una persona distinta da quella che esercita l'ufficio di superiore provinciale ${ }^{10}$. L'intenzione

\footnotetext{
${ }^{10}$ Invece, per quanto riguarda le comunità locali, il can. $636 \$ 1 \mathrm{CIC} / 83$ richiede questa distinzione nella misura possibile. Il diritto proprio potrà definire quando è permesso che l'incarico dell'economo sia svolto dalla persona del superiore. È
} 
del legislatore è non solo di evitare la concentrazione degli incarichi in un'unica persona, ma anche liberare il superiore provinciale dall'amministrazione diretta dei beni temporali della provincia, per renderlo disponibile per altri affari più importanti inerenti al suo ufficio ${ }^{11}$. Non è compito del superiore provinciale di occuparsi di alcune cose dell'amministrazione dei beni che per la loro natura possono essere svolte da altre persone o degli affari di minore importanza che spesso, per essere sbrigati, occuperebbero la maggior parte del tempo a disposizione. Nel caso di concentrazione di questi affari nella persona del superiore provinciale, provocherebbe il rischio che egli trascuri altri settori della provincia che necessitano della sua presenza e del suo intervento come autorità, specialmente nella funzione animatrice della provincia che è direttamente rivolta alle persone ${ }^{12}$.

D'altra parte, l'affidamento dell'amministrazione dei beni temporali della provincia a una persona distinta da quella del superiore, garantisce la sicurezza che l'amministrazione dei beni non sarà trascurata a motivo della concentrazione degli incarichi e degli affari in una stessa persona ${ }^{13}$. Perciò è consigliabile che l'economo provinciale sia libero dallo svolgimento di altre funzioni o incarichi all'interno della provincia.

Nonostante il diritto comune desideri che questi due incarichi siano affidati a persone distinte, è necessario ricordare che non prevede il doppio governo della stessa persona giuridica con compiti separati, ma prevede unità nel governo di tale entità chiamata provincia, nella

da considerare che non è sempre possibile, nella comunità, designare un economo distinto dal superiore, perché la comunità è composta da pochi religiosi o perché, tranne il superiore, non c'è altro religioso adatto a tale funzione. Cf. J.F. CAstaño, Gli istituti di vita consacrata (cann. 573-730), Roma 1995, p. 205.

${ }^{11}$ Cf. E. Gambari, I religiosi nel Codice. Commento ai singoli canoni, Milano 1986, p. 636; V. Mosca, Povertà e amministrazione dei beni negli istituti religiosi, Quaderni di Diritto Ecclesiale 3(1990), p. 244.

${ }^{12}$ Cf. D. Andrés, Le forme di vita consacrata. Commentario teologico-giuridico al Codice di diritto canonico, Roma 2005, p. 254.

${ }^{13}$ Cf. V. De Paolis, Qual è la responsabilità dell'economo e del superiore nell'amministrazione dei bei (cf. c. $636 \$ 1$ )?, Vita Consacrata 27(1991), p. 183. 
persona del superiore provinciale, subordinando ad esso l'economo provinciale ${ }^{14}$. L'economo provinciale esercita il suo compito sotto la direzione del superiore provinciale ${ }^{15}$ con un più o meno preciso intervento del suo consiglio, in modo tale che atti, spese, permessi e pratiche possano essere considerati come compiuti per mandato e col consenso del superiore. Però, il superiore provinciale dovrà rispettare la responsabilità e i suggerimenti dell'economo provinciale, mantenendo con lui uno spirito di fiducia e collaborazione.

L'economo provinciale di solito viene nominato dal superiore provinciale ${ }^{16}$. Il diritto proprio non può trascurare il fatto che l'economo provinciale deve avere le doti che lo definiscono "diligentia

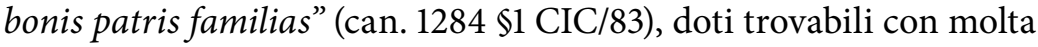
più probabilità in chi appartiene alla famiglia religiosa mediante la professione religiosa. La maggior parte dei canonisti è contraria alla nomina di un laico all'incarico di economo di una struttura religiosa. Anche se non esiste nessuna norma esplicita che proibisce tale scelta, essa tuttavia sarebbe al di fuori e contro la legislazione canonica e da non giustificarsi. L'economo deve operare sotto la responsabilità del superiore e considerando che l'economia è intimamente congiunta con la spiritualità e la missione dell' istituto, essa deve essere amministrata solo da qualcuno che è membro dell'istituto stesso. Questa posizione non pone la questione della fiducia e la valorizzazione dei laici, ma la mancanza della competenza e responsabilità "religiosa" che potrebbe riscontrarsi in una persona che non sia membro dell'istituto ${ }^{17}$.

\footnotetext{
${ }^{14}$ Cf. De Paolis V., L'amministrazione dei beni..., op. cit., p. 121.

${ }^{15} \mathrm{Cf}$. can. $636 \$ 1 \mathrm{CIC} / 83$.

${ }^{16}$ Il diritto proprio, oltre al modo di designazione, deve stabilire non solo la durata dell'ufficio, che di solito coincide con quella del superiore sotto il quale esercita l'ufficio di economo, ma anche le regole di incompatibilità e i modi di cessazione. Cf. D. ANDrÉs, Le forme di vita consacrata..., op. cit., p. 255-256.

${ }^{17}$ Cf. V. De PAolis, Qualche annotazione in margine al documento "Economia e Missione della vita consacrata oggi", Informationes 28/2(2002), p. 125; F. PELoso, Economia, vita consacrata e missione, Informationes 28/1(2002), p. 122; D. ANDRÉs, Le forme di vita consacrata..., op. cit., p. 255; J. KHOURY, Commento al can. 636, in: AA.VV., Commento al Codice di diritto canonico, op. cit., p. 388;
} 
Il secondo aspetto della soggezione dell'incarico dell'economo provinciale, come amministratore dei beni della provincia, all'autorità del superiore provinciale, è il giuramento che egli deve prestare davanti al superiore provinciale, impegnandosi a esercitare il suo incarico con onestà e fedeltà ${ }^{18}$.

L'importanza della responsabilità dell'ufficio di qualsiasi amministratore dei beni temporali, tra cui anche quella dell'economo provinciale, determina il fatto che, all'inizio del loro incarico, si procede, come esige il can. 1283,2 CIC/83, a un accurato e dettagliato inventario di tutti i beni appartenenti alla persona giuridica, che sarà affidata al governo del nuovo amministratore. In questo inventario si dovranno registrare, con una descrizione come prevede il suddetto canone, i beni immobili e mobili, le cose di valore, i beni culturali, i diritti reali e personali e altre cose che appartengono alla persona giuridica. Questo inventario dovrà essere fatto in due copie, come richiesto dal can. 1283,3 CIC/83, in modo tale che una copia sia conservata nell'ufficio dell'amministratore e un'altra nella curia, cioè, nel caso delle province dell'istituto, nella curia provinciale.

Il diritto proprio di ogni istituto, oltre all'elenco dettagliato, previsto dal can. $1284 \$ 2 \mathrm{CIC} / 83$, dei principali obblighi che spettano a tutti gli amministratori dei beni ecclesiastici, deve curare meglio l'attribuzione di tutto ciò che spetta all'economo provinciale e al superiore provinciale nell'amministrazione dei beni della provincia, specialmente definire i casi della subordinazione o dipendenza dell'economo dal superiore provinciale. Il can. $638 \$ 2 \mathrm{CIC} / 83$ concede un principio generale in base al quale tutti gli atti di amministrazione ordinaria sono posti validamente, oltre che dai superiori, anche dagli altri ufficiali designati dal diritto proprio, tra cui gli economi, nei limiti della loro competenza. In questa affermazione non si fa cenno agli atti di amministrazione straordinaria perché la decisione di questi

E. Sastre SAntos, L'affidamento dell'economia di un istituto religioso a persona estranea, Commentarium pro Religiosis et Missionariis 86(2005), p. 312-315.

${ }^{18}$ Cf. can. 1283,1 CIC/83; V. De PAolis, L'amministrazione dei beni..., op. cit., p. 116. 
ultimi spetta al rispettivo superiore con un intervento più o meno determinato del suo consiglio.

Oltre agli atti di amministrazione ordinaria, di cui parleremo più avanti, ci sono anche gli atti più specifici dell'incarico di economo provinciale previsti dal diritto proprio, o quelli che per loro natura devono essere svolti da lui. È necessario affermare che un superiore provinciale che ponesse abitualmente atti di amministrazione ordinaria e quelli specifici, sostituirebbe l'economo provinciale nell'esercizio delle sue funzioni, violando, al tempo stesso, lo spirito e la lettera della norma canonica che affida, in primo luogo, all'economo provinciale l'adempimento di tali atti ${ }^{19}$.

Tocca all'economo provinciale il compito di fornire al superiore provinciale e al suo consiglio, e anche agli stessi religiosi, gli elementi informativi completi per stimolare riflessioni e ricerche su come vivere meglio il valore della povertà e della condivisione dei beni da parte delle diverse componenti della provincia ${ }^{20}$.

All'economo provinciale spetta anche il ruolo di educatore e formatore nell'ambito dell'amministrazione dei beni. Tra le più importanti mansioni di questo settore, c'è quello di accompagnare gli economi locali e gli altri amministratori delle attività e settori della provincia, assicurando loro una formazione adeguata nel settore dell'economia attraverso le consulenze, gli incontri di formazione, con la partecipazioni di diversi specialisti, anche laici. Non dovrà essere trascurata da lui anche la formazione iniziale, offrendo ai religiosi, in questa tappa, incontri sui principi della gestione dei beni temporali secondo il voto di povertà, e la missione della Chiesa e dell'istituto ${ }^{21}$.

\footnotetext{
${ }^{19}$ Cf. V. De PaOlis, La vita consacrata..., op. cit., p. 264; V. Mosca, Povertà e amministrazione..., op. cit., p. 247.

${ }^{20}$ Cf. A. Perlasca, Considerazioni problematiche circa la costituzione di fondazioni civili da parte di istituti religiosi, Informationes 27/1(2001), p. 76.

${ }^{21}$ Cf. Unione Superiori Generali, Economia e missione nella vita consacrata oggi. Documento della 60a assemblea della USG, Roma 2002, p. 28.
} 


\section{Ruolo della commissione provinciale per gli affari economici}

Il diritto comune, al can. $1280 \mathrm{CIC} / 83$, prescrive la costituzione di un consiglio per gli affari economici composto da un gruppo di persone che aiutino il superiore e gli altri amministratori, a norma dei propri statuti, nell'amministrazione dei beni temporali delle persone giuridiche ${ }^{22}$. Senz'altro, la norma del canone è generica e dovrà essere specificata ulteriormente sia circa la composizione, sia circa gli affari da trattare, in base alla normativa del diritto particolare, e specialmente in base agli statuti di tale commissione ${ }^{23}$.

Per quanto riguarda gli istituti religiosi, il legislatore universale già prevede in modo obbligatorio, al can. $626 \$ 1 \mathrm{CIC} / 83$, un consiglio per i superiori religiosi a tutti i livelli. Questo consiglio svolgerà anche le funzioni di consiglio di amministrazione dei beni. Però, questo non esclude che il diritto proprio può prevedere la costituzione di un consiglio per gli affari economici accanto a un vero consiglio del superiore, specialmente quello generale e provinciale e per le strutture equiparate alla provincia ${ }^{24}$.

Gli eventuali commissioni provinciali devono essere create secondo le norme del diritto proprio e delle decisioni particolari del superiore provinciale e suo consiglio. Possono essere composte dai religiosi o laici, esperti nel campo dell'economia, nominati dal superiore provinciale. Come abbiamo accennato, non è esclusa la nomina di un laico esperto in materia economica e pratico della gestione dei

\footnotetext{
${ }^{22}$ Tuttavia, il legislatore ha previsto, nello stesso canone, un'alternativa, cioè al posto di tale commissione è possibile nominare almeno due consiglieri che prestino lo stesso servizio della commissione.

${ }^{23}$ Il Codice vigente, ai cann. 492-493, prevede la costituzione del consiglio diocesano per gli affari economici. I membri, almeno tre chierici o laici, sono nominati dal vescovo diocesano per un quinquennio su base dell'effettiva competenza in economia, in diritto civile, e presuppone una eminente onestà. Tale organismo è presieduto dal vescovo diocesano o da un suo delegato (cf. can. $492 \$ 1 \mathrm{CIC} / 83$ ). Essendo una struttura di controllo e vigilanza, è bene che l'economo non ne faccia parte, ma partecipi alle sedute come relatore. Cf. Conferenza Episcopale ItaLIANA, Istruzione in materia amministrativa (2005), Bologna 2006, nr. 26.

${ }^{24}$ Cf. V. De Paolis, I beni temporali della Chiesa, Bologna 1995, p. 164.
} 
beni temporali negli istituti religiosi. La presenza di tale esperto di fiducia, se non come membro effettivo, almeno come consigliere, secondo il parere dello scrivente è necessaria, considerando che in questa epoca economica la maggior parte delle risorse economiche degli istituti religiosi provengono da investimenti di capitale, ed è importante investire con la necessaria prudenza e accortezza ${ }^{25}$.

Per quanto riguarda le funzioni di tale organismo, esse hanno un carattere piuttosto consultivo, ma importantissimo per la politica della gestione dei beni patrimoniali della provincia.

\section{Amministrazione ordinaria}

Per amministrazione ordinaria dei beni patrimoniali si intende semplicemente quell'amministrazione che spetta in forza dell'ufficio all'economo e riguarda "quegli atti che prevedono la quotidiana amministrazione della persona giuridica e non richiedono altro se non la diligenza del buon padre di famiglia come requisito di realizzabilità"26. Le spese e gli atti giuridici di amministrazione ordinaria, come dispone il can. $638 \$ 2 \mathrm{CIC} / 83$, possono essere effettuati, oltre che dai superiori e dagli economi e dagli altri amministratori inferiori, anche da qualsiasi altro che è stato delegato per i singoli casi dal diritto proprio o dal superiore competente ${ }^{27}$.

Quali siano in concreto tali atti lo si può cogliere particolarmente dal can. $1284 \$ 2 \mathrm{CIC/83}$ e seguenti del vigente Codice, ma adattandoli alla natura e ai fini della persona giuridica. Questi atti

\footnotetext{
${ }^{25}$ Cf. Unione Superiori Generali, Economia e missione..., op. cit., p. 23.

${ }^{26}$ L.Centurioni, L'amministrazione dei beni ecclesiastici: le competenze del vescovo diocesano, Roma 1996, pag. 60; D. ANDRÉs, Le forme di vita consacrata..., op. cit., p. 268-269; A. Vizzali, L'amministrazione dei beni ecclesiastici, in: AA.VV., I beni temporali della Chiesa, Milano 1997, p. 76; J.F. CASTAÑo, Gli istituti di vita consacrata..., op. cit., p. 207.

V. De PAOLIs afferma che "la dottrina canonica ha indicato il concetto di amministrazione ordinaria nei seguenti tre momenti: 1) conservare e tutelare i beni, perché non subiscano danno; 2) far fruttificare i beni; 3) destinare i beni per gli scopi istituzionali". L'amministrazione dei beni..., op. cit., p. 116.

${ }^{27}$ Cf. D. ANDRÉs, Le forme di vita consacrata..., op. cit., p. 271.
} 
di amministrazione ordinaria, integrati da altri, aggiornati ai nostri tempi da alcuni canonisti ${ }^{28}$, sono applicabili a tutti gli amministratori, tra cui anche l'economo provinciale. Essi sono:

- vigilare affinché i beni affidati in qualsiasi modo non vadano distrutti o non subiscano in alcun modo danneggiamenti, assicurando la loro conservazione materiale anche con contratti di assicurazione;

- tutelare la proprietà dei beni temporali in forma civilmente valida;

- impedire, con la disposizione di diversi mezzi, l'usurpazione o la scomparsa dei beni mobili o immobili;

- osservare le norme del diritto canonico e civile, e le prescrizioni imposte dalla legittima autorità, come pure le disposizioni dei fondatori e donatori, e badare che non derivi dalla loro inosservanza danno alla Chiesa, ossia alla persona giuridica;

- provvedere alla manutenzione ordinaria degli immobili e alle debite garanzie di efficienza dei beni amministrati;

- riscuotere a tempo debito i redditi e i proventi, che devono essere conservati o impiegati secondo le intenzioni del fondatore o donatore, o le prescrizioni e le norme del diritto;

- pagare, nel tempo stabilito, gli interessi dovuti a causa di un mutuo o di un'ipoteca, e curare opportunamente la restituzione del capitale;

- pagare le altre forme di utenze e di imposte fiscali;

- distribuire gli stipendi ai dipendenti;

- vendita dei prodotti agricoli e di allevamento;

- impiego delle eccedenze monetarie per i fini dell'istituto, dopo aver concordato con il rispettivo superiore;

- tenere ben ordinati i libri delle entrate e delle uscite;

\footnotetext{
${ }^{28}$ Cf. V. De Paolis, L'amministrazione dei beni..., op. cit., p. 117; L. CENTURIONI, Amministrazione dei beni ecclesiastici..., op. cit., p. 60-61; L. Mistó, I beni temporali della Chiesa (cann. 1254-1310), in: AA.VV., Il diritto nel mistero della Chiesa, vol. III, Roma 1992, p. 403-404.
} 
- compilazione e presentazione del bilancio consultivo annuale, e di quello preventivo, se richiesto;

- accurata catalogazione e custodia di tutti i documenti relativi ai beni della persona giuridica;

- fornire agli altri amministratori le informazioni necessarie vincolate alla gestione dei beni temporali;

- aggiornare l'inventario dei beni affidati all'amministrazione, secondo le norme del diritto e le disposizioni dei superiori;

- fare gli acquisti necessari per la conduzione dell'attività della persona giuridica.

Questo elenco degli atti di amministrazione ordinaria, che mai si esaurisce, può essere completato in parte dal diritto proprio dell' istituto o dagli statuti della persona giuridica che in maniera più specifica fanno la distinzione tra gli atti di amministrazione ordinaria e quelli di amministrazione straordinaria.

Oltre agli atti di amministrazione ordinaria, affidati agli economi a diversi livelli della struttura dell'istituto, raccolti nel dettato del can. $1284 \mathrm{CIC} / 83$, ci sono molti altri atti di amministrazione ordinaria, talora inseriti nella comune gestione dei beni della provincia, secondo la tecnica amministrativa conforme ai fini di queste entità, agli usi del nostro tempo e alle leggi dello Stato nei quali tale entità disimpegna il suo operato. Spesso la normativa e la dottrina canonica non ha per loro il debito interesse, perché il loro operato richiede, di solito, un intervento discrezionale dell'autorità competente, ma viene adempiuta con prudenza, povertà, giustizia e spesso in dialogo reciproco tra l'economo e il superiore provinciale.

Nel campo dell'amministrazione ordinaria è da collocare anche l'amministrazione ordinaria di maggiore importanza che non dovrebbe essere considerata come amministrazione straordinaria. La distinzione degli atti di maggior importanza dagli atti di amministrazione ordinaria è accennata dal can. $1277 \mathrm{CIC} / 83$, che si riferisce all'amministrazione dei beni della diocesi, ma che può essere 
validamente adottata anche per gli istituti religiosi ${ }^{29}$. Gli atti di amministrazione ordinaria di maggior importanza non necessariamente sono riservati all'autorità della persona giuridica, ma possono essere posti anche dagli amministratori, cioè nel caso degli istituti religiosi, anche dagli economi ai diversi livelli, ma seguendo una peculiare procedura che di solito richiede di sollecitare il parere di qualcuno o avere qualche approvazione. In concreto, per certi atti di maggior importanza, anche se rientrano nell'amministrazione ordinaria, l'economo dovrebbe ascoltare il parere della commissione per gli affari economici o, prima di compierli, dovrebbe consultare, oppure ottenere l'approvazione del proprio superiore provinciale.

\section{Amministrazione straordinaria}

Partendo dal can. $1281 \$ 1 \mathrm{CIC} / 83$, che tuttavia appare molto stringato e generico, possiamo dire che l'amministrazione straordinaria è quella che sorpassa i limiti e i modi dell'amministrazione ordinaria, e i cui atti, per essere compiuti validamente, richiedono una specifica procedura ${ }^{30}$. Questi atti non rientrano quindi nell'ambito della competenza autonoma dell'economo come amministratore, perché, considerando la gravità dell'atto, superano la soglia di normalità quotidiana che è tipica dell'ordinarietà dell'amministrazione ${ }^{31}$. Il ruolo

29 Cf. V. De Paolis, L'amministrazione dei beni..., op. cit., p. 118; A. Vizzari, L'amministrazione dei beni ecclesiastici..., op. cit., p. 77-78.

${ }^{30}$ I controlli canonici previsti per gli atti di amministrazione straordinaria sono di tre tipi:

- la licenza, che spesso viene denominata anche come autorizzazione, permesso o nulla osta, concessa in forma scritta dalla competente autorità ecclesiastica cui la persona giuridica è soggetta;

- consenso da parte di un organo indicato dal diritto, necessario per la validità dell'atto; - il parere che deve essere richiesto nonostante esso non abbia carattere vincolante, per la validità dell'atto, a coloro che sono indicati dal diritto. Cf. Conferenza Episcopale Italiana, Istruzione in materia amministrativa (2005)..., op. cit., nr. 60; D. ANDrÉs, Le forme di vita consacrata..., op. cit., p. 270-271.

${ }^{31}$ Cf. L. Centurioni, Amministrazione dei beni ecclesiastici..., op. cit., p. 67; V. DE Paolis, L'amministrazione dei beni..., op. cit., p. 118; A. Vizzari, L'amministrazione dei beni ecclesiastici..., op. cit., p. 78-82. 
dell'economo o di qualsiasi altro amministratore si limita a essere quello di esecutore delle decisioni prese dai superiori competenti. Per compiere validamente questi atti, essi devono ottenere per iscritto, come dispone il can. $1281 \$ 1 \mathrm{CIC/83}$, la debita facoltà del superiore competente.

Il Codice vigente, nella formulazione dell'amministrazione straordinaria, è molto generico, offrendo soltanto un criterio generale di tale amministrazione che consiste nel superamento del fine e modo dell'azione amministrativa ordinaria dell'amministratore. A questi due criteri, alcuni canonisti ne aggiungono altri per individuare se un atto sia o diventi straordinario: valore della cosa, quantità, rischi di perdita, incidenza dell'atto sui frutti, pericoli di instabilità del patrimonio, natura della casa, oggetto dell'atto di amministrazione e del servizio che viene prestato, modalità e complessità delle trattative, durata dei tempi di esecuzione, incertezza dei risultati economici, situazione patrimoniale ed economica della stessa persona giuridica ${ }^{32}$.

Sulla base di questi criteri, gli istituti religiosi, nel loro diritto proprio, possono determinare altri criteri che permettano loro di stabilire, come richiede il can. $638 \$ 2 \mathrm{CIC} / 83$, gli atti di amministrazione straordinaria $^{33}$, sia quale debba essere il soggetto competente per ap-

${ }^{32}$ Cf. V. De Paolis, I beni temporali della Chiesa..., op. cit., p. 146; L. Centurioni, L'amministrazione dei beni ecclesiastici..., op. cit., p. 68.

${ }^{33}$ Il prof. D. ANDRÉs fa un elenco generale degli atti di amministrazione straordinaria per gli istituti religiosi:

"1) alienazione stretta, in tutte le sue specie;

2) ogni negozio giuridico che peggiori la condizione patrimoniale della rispettiva persona giuridica;

3) operazioni bancarie fisse, investimenti ad alto rischio o che immobilizzino per un tempo eccessivo il capitale;

4) copertura e pagamento di debiti ingenti;

5) collocazione che implichi alienazione;

6) ogni tipo di spese, in generale, non giustificate dai fini di manutenzione e funzionamento normali o ordinari delle cose". Le forme di vita consacrata..., op. cit., p. 270. E da sottolineare che il Codice del 1983, nel can. $1254 \$ 1$, distingue l'alienazione dagli atti di amministrazione straordinaria, anche se l'alienazione in sé è comunque un atto straordinario, però la natura di tali atti è diversa, come anche la 
porli, come pure la procedura da applicare. In realtà, il diritto proprio degli istituti religiosi dovrà determinare a chi spetta la competenza per gli atti di amministrazione straordinaria tra i vari superiori, con un determinato intervento del loro consiglio.

Nel diritto possono essere considerati come atti dell'amministrazione straordinari:

- prestare o affittare dei beni, o cederne alcuni diritti parziali in modo che il possesso, l'uso e l'usufrutto sono concessi per un tempo a un'altra persona, giuridica o fisica, estranea all'istituto;

- gravare certi beni di una pensione o di un canone annuo e con ciò lasciare ad altri le rendite della proprietà;

- impegnare o ipotecare determinati beni immobili, o darli in garanzia, in modo che un eventuale diritto di proprietà sia ceduto ad altri in caso di mancato pagamento di un debito;

- contrarre, in qualsiasi forma, debiti e obbligazioni, di qualsiasi sorta, con o senza garanzie;

- contrarre debiti e il trasferimento di beni tra le persone giuridiche della Congregazione.

Tutti questi atti non rientrerebbero nell'ambito di competenza dell'economo, ma sarebbero riservati al superiore competente, con il consenso del proprio consiglio, nei limiti della propria competenza.

Prendendo tutto questo in considerazione, il superiore provinciale, con il consenso del suo consiglio, può compiere validamente tutti gli atti di amministrazione straordinaria quando il valore dei beni a cui questi atti si riferiscono, o la somma di qualsiasi altro negozio, non supera il limite fissato per l'amministrazione straordinaria dei beni patrimoniali dal superiore generale per una determinata provincia. In questo contesto entrano nella competenza del superiore provinciale

regolamentazione. L'amministrazione, sia ordinaria che straordinaria, riguarda gli atti che toccano i beni che rimangono come beni della persona dopo tali interventi; invece, l'alienazione è un atto che fa uscire il bene dal patrimonio della persona giuridica. L'amministrazione straordinaria riguarda tutti i beni della persona giuridica, invece l'alienazione solo quelli che appartengono al patrimonio stabile. Cf. V. De Paolis, L'amministrazione dei beni..., op. cit., p. 119-120. 
tutti gli atti dell'amministrazione straordinaria dei beni patrimoniali delle comunità locali e delle altre persone giuridiche soggette alla sua autorità che superano la competenza della comunità per atti di tale amministrazione, ma non oltrepassano il limite stabilito per rispettiva provincia. Invece, nel caso che gli atti di amministrazione straordinaria dei beni patrimoniali superino la competenza del superiore provinciale, per compierli validamente, occorre ricevere l'autorizzazione del superiore generale.

Per ottenere l'autorizzazione per compiere gli atti di amministrazione straordinaria dei beni patrimoniali che oltrepassano la competenza del superiore provinciale, quest'ultimo dovrà trasmettere al superiore generale la seguente documentazione, richiesta nella prassi di tanti istituti religiosi:

- domanda scritta nella quale è presentato, in maniera dettagliata, l'atto di amministrazione straordinaria dei beni patrimoniali da compiere, nel contesto della situazione patrimoniale ed economica della comunità e provincia, con una appropriata motivazione;

- parere o consenso del consiglio provinciale, con l'estratto del verbale della seduta del consiglio provinciale, e della comunità locale coinvolta, se necessario;

- perizia fatta da un'entità civile circa il valore del bene che è oggetto dell'atto di amministrazione straordinaria;

- nel caso della contrattazione dei debiti, nella domanda dovranno essere segnalati tutti gli altri debiti e obbligazioni che attualmente gravano sulla casa religiosa e la provincia. Inoltre, il richiedente dovrà dimostrare che le normali entrate della propria attività permetteranno di pagare gli interessi e di ammortizzare gradualmente il capitale secondo le intese in un periodo di tempo ragionevole ${ }^{34}$.

Però, alcuni di questi atti, se l'importo supera la somma stabilita dalla Santa Sede per le singole nazioni e, al tempo stesso, comportano un pericolo di danneggiamento della situazione economica della

${ }^{34}$ Cf. can. $639 \$ 1$ CIC/83. 
provincia o istituto, necessitano dell'autorizzazione della Santa Sede stessa $^{35}$. A questi atti o negozi si aggiungono, come dispone il can. $638 § 3$, le donazioni votive fatte alla Chiesa e le cose preziose per valore artistico o storico.

Il superiore generale, in tutti questi casi di atti dell'amministrazione straordinaria dei beni patrimoniali che superano la sua competenza, deve inoltrare tutta la documentazione preparata dal superiore provinciale alla Santa Sede per ottenere l'autorizzazione, aggiungendo il proprio parere e quello del consiglio.

\section{Alienazione dei beni}

Gli atti di alienazione, come è stato già detto precedentemente, sono stati distinti dall'attuale legislazione canonica, al can. $1254 \$ 1$ CIC/83, dagli atti dell'amministrazione straordinaria anche se di per sé l'atto di alienazione è un atto straordinario nella vita della provincia di un istituto. Questa distinzione conferma anche la distinta normativa per gli atti di amministrazione straordinaria contenuta nel can. 1281 CIC/83 e quella per l'alienazione collocata nei cann. 1290-1298 $\mathrm{CIC/83}$. Nel caso degli istituti religiosi, essa viene completata dal can. $638 \$ 3 \mathrm{CIC} / 83$. Tutte queste norme sono ulteriormente arricchite dal diritto proprio di ogni istituto, il quale, con norme proprie, rende applicabili le disposizioni del diritto comune alla situazione concreta dell'istituto.

Il concetto di alienazione consiste nel trasferimento del diritto di proprietà di beni, appartenenti al patrimonio stabile, a un'altra persona giuridica o fisica estranea all' istituto ${ }^{36}$. Questo trasferimento avviene, per esempio, con una vendita, una donazione o una permuta.

\footnotetext{
${ }^{35}$ Cf. can. $638 \$ 3 \mathrm{CIC} / 83$. Il prof. D. ANDRÉs afferma che questi atti sono segnalati con i verbi: alienari (alienazione), pignorari (dare in prestito - impegnare), hypothecae nomine obligari (ipotecare), locari (collocazione), emphyteusi redini (enfiteusi o cessione del dominio utile in cambio di una vendita), aes alienum contrahere (debiti). Le forme di vita consacrata..., op. cit., p. 273-274.

${ }^{36}$ Cf. J.J. Pineda, La capacidad económica de institutos..., op. cit., p. 110; L. Mistó, I beni temporali della Chiesa..., op. cit., p. 406.
} 
Tale definizione include nell'alienazione anche la distruzione volontaria di un bene appartenente al patrimonio stabile.

E da sottolineare che il vigente Codice equipara, per quanto riguarda la procedura, all'alienazione in senso proprio anche quella in senso improprio, qualsiasi negozio per il quale la situazione patrimoniale della persona giuridica potrebbe subire detrimento ${ }^{37}$.

I beni, che per legittima assegnazione appartengono al patrimonio stabile della persona giuridica, non possono essere validamente alienati senza la debita licenza scritta dell'autorità competente ${ }^{38}$. Nel caso degli istituti religiosi, il concedere questa facoltà spetta, come afferma il can. $638 \$ 3 \mathrm{CIC} / 83$, al competente superiore, sempre con il consenso del proprio consiglio, a condizione che il valore dei beni non superi la somma stabilita dall'autorità competente ${ }^{39}$.

L'assegnazione della competenza per concedere la licenza in vista dell'alienazione di beni patrimoniali è regolata dalle norme di diritto proprio di ogni istituto. Secondo queste norme i superiori delle province spetta la facoltà di concedere la licenza, con il consenso del suo consiglio, per alienare beni propri appartenenti al patrimonio stabile delle rispettive entità il cui valore supera la somma stabilita da questi superiori per le rispettive comunità come limite dell'amministrazione straordinaria dei beni patrimoniali e loro alienazione, a condizione

${ }^{37}$ Difatti, al can. $638 \$ 3$ il legislatore equipara all'alienazione ad altri quattro negozi giuridici:

"1) qualsiasi negozio in cui la condizione patrimoniale della persona giuridica interessata corra il rischio di peggiorare;

2) qualsiasi negozio in cui l'importo superi la quantità massima prefissata dalla Santa Sede per ogni regione;

3) qualsiasi negozio in cui siano implicate donazioni votive alla Chiesa;

4) qualsiasi negozio in cui si tratti di oggetti preziosi per motivi artistici o storici”. D. ANDrés, Le forme di vita consacrata..., op. cit., p. 272; cf. V. DE PAOLIS, L'amministrazione dei beni..., op. cit., p. 120; F.J. EgAÑA, Quali elementi devono essere contenuti nella richiesta di alienazione di beni ecclesiastici?, Vita Consacrata 27(1991), p. 986.

${ }^{38}$ Cf. can. 1291 CIC/83.

${ }^{39}$ Cf. D. AndrÉs, Le forme di vita consacrata..., op. cit., p. 274-275; J.F. CAstAÑo, Gli istituti di vita consacrata..., op. cit., p. 205-206. 
che il valore di questo bene da alienare non superi la somma fissata dal superiore generale per la rispettiva provincia. Invece, nel caso che il valore di questo bene patrimoniale superi la competenza del superiore provinciale, ma non superi i limiti fissati dalla Conferenze Episcopali del luogo e approvati dalla Santa Sede, la competenza di concedere la licenza di procedere all'alienazione passa al superiore generale che, per concederla, dovrà ottenere il consenso del suo consiglio. Nel caso che il valore del bene da alienare superi la competenza del superiore generale occorre ottenere, per la validità di questo atto, la licenza della Santa Sede.

Il superiore provinciale, per ottenere dal superiore generale licenza ai fini di procedere all'alienazione di un bene che fa parte del patrimonio stabile della stessa provincia o di una comunità, dovrà inoltrare al superiore generale la richiesta dalla quale risulti, in modo chiaro, che la volontà di alienare il bene è stata supportata, come dispone il can. $1293 \$ 1,1 \mathrm{CIC} / 83$, da una giusta e seria causa, valutata attentamente dallo stesso superiore provinciale e il suo consiglio. A tale richiesta, che in modo dettagliato descrive il bene da alienare ${ }^{40}$, e i motivi per cui si deve procedere all'alienazione e alcuni dettagli della stessa alienazione, tra cui il possibile acquirente e la somma di transazione. Nella prassi di diversi istituti si dovrà allegare, anche:

- il parere della comunità, se il bene da alienare appartiene a una comunità della provincia;

- il parere dell'economo provinciale e quello della commissione provinciale per gli affari economici, se tale è stata costituita nella provincia;

- l'estratto del verbale del consiglio provinciale nel quale deve figurare il consenso del consiglio provinciale per procedere all'alienazione;

\footnotetext{
${ }^{40}$ Se si tratta di alienare un bene divisibile, di cui una parte sia già stata alienata, la richiesta per ottenere l'autorizzazione, sotto la pena di invalidità della stessa alienazione, dovrà menzionare le parti già alienate (cf. can. $1292 \$ 3 \mathrm{CIC} / 83$ ).
} 
- una perizia scritta, possibilmente convalidata davanti all'organo civile competente, che attesta il valore del bene che è oggetto di alienazione ${ }^{41}$;

- il parere dell'ordinario del luogo ove è ubicato il bene oggetto della progettata alienazione ${ }^{42}$.

È da sottolineare che il superiore provinciale, per concedere, nei limiti della sua competenza, l'autorizzazione di procedere all'alienazione di beni appartenenti alle persone giuridiche soggette alla sua autorità, deve ricevere ed esaminare la documentazione nella forma sopra esposta.

Invece, nel caso in cui il superiore generale non ha la competenza di concedere la licenza di alienazione dei beni della rispettiva provincia perché il loro valore oltrepassa i limiti della sua competenza, egli dovrà inoltrare la pratica preparata dal superiore provinciale alla Santa Sede, allegando ad essa il proprio parere e quello del consiglio generale.

${ }^{41}$ Cf. can. 1293; F.J. EgAÑA, Quali elementi..., op. cit., p. 989.

Da notare nel can. $1293 \$ 1,2 \mathrm{CIC} / 83$, due osservazioni: si parla di periti al plurale e che la perizia deve essere rilasciata per iscritto. Questa è la regola di prudenza, afferma J.F. CAstaño, che permette di evitare la possibilità che un solo perito si sbagli nella valutazione. Se esiste una discordanza nella valutazione, è opportuno chiedere un terzo parere. Cf. Gli istituti di vita consacrata..., op. cit., p. 222.

$\mathrm{Nel}$ caso che la somma offerta per la progettata alienazione sia inferiore a quella testata nella perizia, nella richiesta il superiore provinciale è obbligato a motivare tale situazione.

${ }^{42}$ Benché la legislazione canonica del Codice vigente non prescriva questo requisito per gli istituti di vita consacrata e le società di vita apostolica di diritto pontificio, la CIVCSVA, pur riconoscendo la piena autonomia di governo a tali istituti, i quali possono disporre liberamente dei propri beni, sempre nel rispetto della legge canonica e delle finalità dell'istituto ha introdotto, nello spirito del can. $1293 \$ 2 \mathrm{CIC} / 83$, la prassi di obbligare questi istituti a informare l’ordinario del luogo ove è ubicato il bene che si intende alienare. Cf. CIVCSVA, Lettera del segretario della Congregazione ai/alle superiori/e generali del 21.12.2004, Prot. nr 971/2004, p. 2. I motivi di tale prassi sono contenuti nella stessa lettera: "per favorire le mutue relazioni tra vescovi e istituti, e per evitare, nei limiti del possibile, che il patrimonio ecclesiastico si impoverisca, ed infine per consentire all'ordinario del luogo di valutare l'opportunità dell'acquisto del bene". 


\section{Conclusione}

Ogni provincia di qualsiasi istituto religioso, come persona giuridica della Chiesa, ha diritto di acquistare, possedere, amministrare e alienare i beni temporali. Questi negozi possono essere compiuti dai superiori provinciali secondo le disposizioni del diritto universale e quello proprio dell'istituto religioso. Il superiore provinciale può porre tutti gli atti di amministrazione ordinaria nel rispetto dei compiti dell'economo provinciale. Ponendo questi atti bisogna stare attenti a non creare conflitti. Per questo, tra superiore ed economo occorrono dialogo continuo, rispetto reciproco e fiducia vicendevole. Invece gli atti della amministrazione straordinaria possono essere effettuati solo dal superiore provinciale, che ha la facoltà di delegare questo diritto ad altri religiosi, compresso l'economo provinciale. Per quanto riguarda l'atto di alienazione, il superiore provinciale non può porre tale atto, se il valore del bene da alienare superi la somma fissata dal superiore generale per la rispettiva provincia. $\mathrm{Nel}$ caso, che valore di questo bene patrimoniale superi i limiti fissati dalla Conferenza Episcopale del luogo e approvati dalla Santa Sede occorre ottenere, per la validità di questo atto, la licenza della Santa Sede. Oltre di tutto questo, il superiore ha il diritto-dovere di vigilare sull'amministrazione dei beni della provincia e di tutte le persone giuridiche soggette alla sua autorità. Questa vigilanza viene attuata tramite dialogo comunitario, gli organi di consultazione permanente (Commissione Economica) o di consultazioni ad hoc (costituiti per i problemi particolari), pareri o consensi del proprio consiglio e il dialogo constante con l'economo provinciale.

Il superiore provinciale e tutti gli amministratori devono vigilare alla conservazione e, all'occorrenza, all'aumento dei beni patrimoniali poiché questi costituiscono la base materiale della vita, dell'attività e dello sviluppo della Congregazione. 


\section{Competence of the provincial superior of a religious institute in the field of material goods and their administration}

Each province of the religious institutes, as a legal person in the Church has the right to acquire, possess, administer and alienate their material goods. These acts are taken by the provincial superiors while maintaining the common law and of own institute. All activities related to the ordinary administration may be taken by those superiors, but respecting the roles and responsibilities of the Provincial Treasurers provided for by law in this regard. Hence, fulfilling the acts of ordinary administration of material goods we should avoid any conflict between the two offices. Between the superior and treasurer there should be a mutual trust and be kept a constant dialogue on the administration of the entrusted material goods. Any acts of extraordinary administration remain within the competence of the provincial superior, who in some cases provided by law, may/can delegate them to other members of the Institute, including treasurers. While the act of alienation of material goods can be taken by the provincial superior or general, depending on the value of alienated goods. If the value of the transaction exceeds the sum determined by the Holy See for the region, then the permission for the act of alienation shall be given by the Holy See.

PAROLE-CHIAVE: provincia, superiore provinciale, beni temporali, economo provinciale, amministrazione ordinaria, amministrazione straordinaria, alienazione

KEY WORDS: province, provincial superior, temporal goods, provincial treasurer, ordinary administration, extraordinary administration, alienation

\section{Nota o Autorze:}

Ks. DR hab. MAREK Stokeosa SCJ, - pracownik naukowo-dydaktyczny na Wydziale Prawa Kanonicznego Uniwersytetu Kardynała Stefana Wyszyńskiego w Warszawie (UKSW); adiunkt w Katedrze Prawa o Posłudze Uświęcania; prodziekan Wydziału Prawa Kanonicznego UKSW; członek Stowarzyszenia Kanonistów Polskich oraz Consociatio Internationalis Studio Iuris Canonici Promovendo. 